\title{
Fragmentation of Oligoribonucleotides from Gas-Phase Ion-Electron Reactions
}

\author{
Jiong Yang and Kristina Håkansson \\ Department of Chemistry, University of Michigan, Ann Arbor, Michigan, USA
}

\begin{abstract}
We have recently demonstrated that both electron capture dissociation (ECD) and electron detachment dissociation (EDD) can provide complementary sequence-specific cleavage of DNA compared with collision activated dissociation (CAD) and infrared multiphoton dissociation (IRMPD). However, EDD is preferred because of more extensive fragmentation at higher sensitivity (due to its negative ion mode operation). Here, we extend the radical ion chemistry of these two gas-phase ion-electron reaction techniques to the characterization of RNA. Compared with DNA, rather limited information is currently available on the gas-phase fragmentation of RNA. We found that the ECD fragmentation patterns of the oligoribonucleotides $\mathrm{A}_{6}, \mathrm{C}_{6}$, and CGGGGC are nucleobase dependent, suggesting that cleavage proceeds following electron capture at the nucleobases. Only limited backbone cleavage was observed in ECD. EDD, on the other hand, provided complete sequence coverage for the RNAs $A_{6}, C_{6}$, $\mathrm{G}_{6}, \mathrm{U}_{6}, \mathrm{CGGGGC}$, and GCAUAC. The EDD fragmentation patterns were different from those observed with CAD and IRMPD in that the dominant product ions correspond to $d$ - and $w$-type ions rather than $c$ - and $y$-type ions. The minimum differences between oligoribonucleotides suggest that EDD proceeds following direct electron detachment from the phosphate backbone. (J Am Soc Mass Spectrom 2006, 17, 1369-1375) @ 2006 American Society for Mass Spectrometry
\end{abstract}

$\mathrm{D}$ eoxyribonucleic acid (DNA) and ribonucleic acid (RNA) play several important roles in a variety of biological processes, including transcription and translation. RNA molecules are unique biopolymers in that they can both carry genetic information and catalytic function [1-3]. As for proteins, RNA function is structurally related and methods for sensitive and detailed structural characterization are needed. NMR spectroscopy is widely used for RNA structural analysis $[4,5]$ but suffers from limited sensitivity. Another powerful approach is chemical labeling followed by fluorescence resonance energy-transfer (FRET) measurements [6], which can provide single molecule sensitivity [7]. However, structural information from FRET is limited to distance constraints for the added fluorophores and does not provide a detailed picture.

McCloskey and coworkers pioneered mass spectrometric analysis of nucleic acids [8,9] and have applied LC-MS to the interrogation of RNA reaction mechanisms and to the characterization of RNA modifications [10-12]. Tandem mass spectrometry (MS/MS) is widely used to characterize oligonucleotides and PCR products [13-18]. Particularly, dissociation of even-electron oli-

Published online July 26, 2006

Address reprint requests to Dr. K. Håkansson, Department of Chemistry, University of Michigan, 930 North University Ave., Ann Arbor, MI 481091055, USA. E-mail: kicki@umich.edu godeoxynucleotide (DNA) anions with "slow heating" techniques, including nozzle-skimmer dissociation [19], collision activated dissociation (CAD) [20], infrared multiphoton dissociation (IRMPD) [21], and multipole storage-assisted dissociation [22] produces sequencespecific $[a-\mathrm{B}]$ and $w$-type ions (McLuckey nomenclature [23]) from cleavage of the phosphodiester backbone. With those techniques, nucleobase loss alone is also observed as a major fragmentation pathway. Such ions are not desired because they do not provide sequence-specific information. We have shown that irradiation of gas-phase oligodeoxynucleotide dications with low-energy $(<0.2 \mathrm{eV})$ electrons (electron capture dissociation (ECD) [24-27]) provides complementary sequence-specific backbone cleavage with the major product ions being $w$ and $d$ ion series (corresponding to cleavage of carbon-oxygen bonds at different sides of an inter-residue phosphate group [23]). In addition, radical $a / z$ as well as $(a / z-B)$ and $(c / x-B)$ ions are produced but nucleobase loss constitutes a minor fragmentation pathway $[28,29]$. ECD has also been shown to be "soft" enough to retain gas-phase hydrogen bonding in $\mathrm{dA}_{6}$ (similar to protein ECD [30]), thereby allowing characterization of higher order structure [29]. However, because ECD requires cationic precursor ions, its sensitivity for nucleic acid characterization is limited. Electron detachment dissociation (EDD), involving irradiation with $\geq 10 \mathrm{eV}$ electrons, was recently introduced by Zubarev and coworkers as a means to evoke radical ion 
mediated fragmentation pathways for biomolecular anions [31]. We have demonstrated that EDD can provide extensive backbone fragmentation of oligodeoxynucleotides at higher sensitivity than ECD [32]. In addition, EDD can cleave backbone covalent bonds without rupturing noncovalent interactions, thereby having potential for characterizing higher order structure.

RNA oligonucleotides (oligoribonucleotides) have been much less characterized with MS/MS and their fragmentation pathways are not as well known. A couple of investigations have shown that low-energy CAD results primarily in abundant $c$-type ions and their complementary $y$-type ions as the major sequence ions $[33,34]$, and a similar pattern was observed with IRMPD [21]. Here, we present the fragmentation pathways in ECD and EDD of hexamer oligoribonucleotides and discuss the utility of these two gas-phase ionelectron reaction techniques for structural characterization of RNA.

\section{Experimental}

\section{Sample Preparation}

Anion-exchange and high-performance liquid chromatography purified $\mathrm{A}_{6}, \mathrm{C}_{6}, \mathrm{G}_{6}$, CGGGGC and GCAUAC, and polyacrylamide gel electrophoresis purified $\mathrm{U}_{6}$ ammonium salts from TriLink BioTechnologies, Inc. (San Diego, CA) were used without further purification. Negative mode electrospray solvent consisted of 1:1 (vol/vol) isopropanol:water (Fisher, Fair Lawn, NJ) with $10 \mathrm{mM}$ ammonium acetate (Fisher). Positive mode electrospray solvent consisted of 1:1 (vol/vol) acetonitrile:water (Fisher) with $0.5 \%$ formic acid (ACROS Organics, Fair Lawn, NJ). The final oligoribonucleotide concentration was 5 to $20 \mu \mathrm{M}$ with the exception of $\mathrm{G}_{6}$, which was electrosprayed at $100 \mu \mathrm{M}$.

\section{Fourier Transform Ion Cyclotron Resonance Mass Spectrometry}

All experiments were performed with a 7 tesla quadrupole-Fourier transform ion cyclotron resonance (Q-FT-ICR) mass spectrometer (Bruker Daltonics, Billerica, MA), which has been previously described [32]. Briefly, samples were infused via an external Apollo electrospray ion source at a flow rate of $70 \mu \mathrm{L} / \mathrm{h}$, mass selectively externally accumulated for 1-10 s (except for the oligonucleotide $G_{6}$, which was accumulated for $60 \mathrm{~s}$ ) and captured in the ICR cell by dynamic trapping. The accumulation, ion transfer, and capture events were looped twice for improved precursor ion abundance. For ECD experiments, further isolation was accomplished by correlated harmonic excitation fields (CHEF) [35]) inside the ICR cell. ECD and EDD were performed before the trapping plate voltages were ramped down. The initial trapping voltage was $-2 \mathrm{~V}$ and the voltage during excitation and detection was $-1 \mathrm{~V}$. An indi-

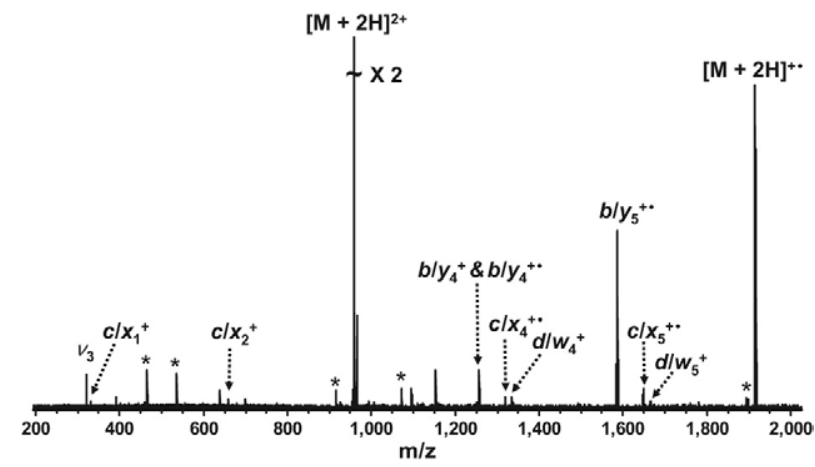

Figure 1. ECD ( $-1 \mathrm{~V}$ bias voltage, $80 \mathrm{~ms}$ irradiation, 32 scans) of the RNA $\mathrm{A}_{6} . b / y, c / x$, and $d / w$ ions are observed in addition to an abundant charged reduced radical species, $[\mathrm{M}+2 \mathrm{H}]^{+}$. Electronic noise spikes are labeled with asterisks.

rectly heated hollow dispenser cathode (previously described [32]) was used to generate electrons for ECD and EDD. During the EDD event, the cathode voltage was pulsed to $-18 \mathrm{~V}$ for $2 \mathrm{~s}$. A lens located in front of the cathode was kept at $-19 \mathrm{~V}$ throughout the experiment. For ECD, the cathode voltage was pulsed to $-1 \mathrm{~V}$ for $35-80 \mathrm{~ms}$. The lens electrode was kept at $+1 \mathrm{~V}$. All mass spectra were acquired with the XMASS software (Bruker) in broadband mode from $\mathrm{m} / \mathrm{z} 200$ to 2000 with $256 \mathrm{k}$ data points and summed over 32-50 scans. The calculated masses of the doubly charged precursor ions and the charge-reduced species were used for internal calibration of all spectra, except for EDD of $A_{6}$ and $U_{6}$, which did not produce the latter species. In those two cases, the $w_{5} / d_{5}$ product ion was used instead. Product ions were assigned based on a $15 \mathrm{ppm}$ error criterion.

\section{Results and Discussion}

\section{Electron Capture Dissociation of $A_{6}$ Dications}

Figure 1 shows an ECD spectrum of the doubly protonated RNA $\mathrm{A}_{6}$. The most abundant product ion in the spectrum corresponds to the charge-reduced species, which has captured an electron but not dissociated. The isotopic distribution of this singly charged ion indicates that it is mainly a radical ion, $[\mathrm{M}+2 \mathrm{H}]^{+}$, i.e., no significant hydrogen loss (or gain) to produce an evenelectron species is seen [36]. The $[\mathrm{M}+2 \mathrm{H}]^{+\cdot}$ species was also dominant in ECD of $\mathrm{dA}_{6}[29]$ in contrast to other oligodeoxynucleotides, which dissociated more readily. The greater stability of $\mathrm{A}_{6}$ and $\mathrm{dA}_{6}$ charge-reduced species is attributed to the presence of intramolecular hydrogen bonds between the adenine bases and the phosphate backbone, preventing product ions from separating and thereby from being detected. Such intramolecular gas-phase hydrogen bonding was previously proposed from both molecular modeling and gas-phase hydrogen exchange experiments [37, 38].

In addition to the abundant charge-reduced radical species, several $b / y, c / x$, and $d / w$ product ions are 


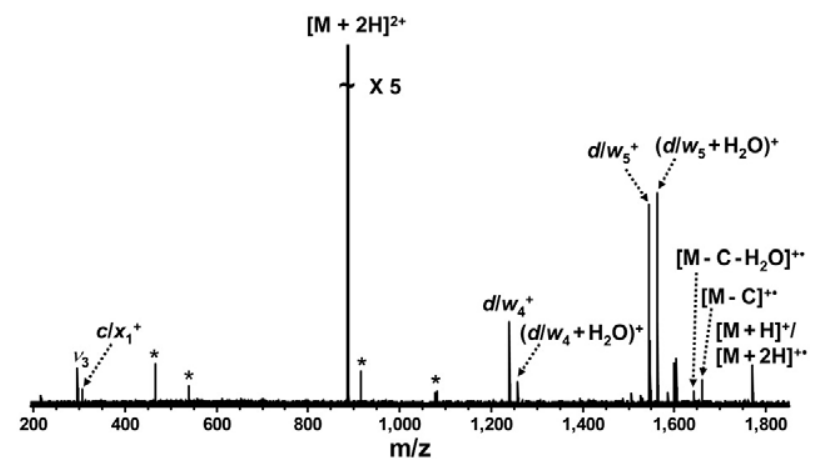

Figure 2. ECD ( $-1 \mathrm{~V}$ bias voltage, $80 \mathrm{~ms}$ irradiation, 32 scans) of the RNA $\mathrm{C}_{6}$. The most abundant products correspond to $w / d$ and $\left(w / d+\mathrm{H}_{2} \mathrm{O}\right)$ ions. Electronic noise spikes are labeled with asterisks.

observed in ECD of $\mathrm{A}_{6}$ (these ion pairs cannot be distinguished due to identical mass). The most abundant of these are radical ions, i.e., they contain one additional hydrogen atom compared with their evenelectron counterparts. However, a complete ion series is not observed for either ion type. In low-energy CAD and IRMPD of oligo RNA, $y$ and c-type ions are the most abundant products in addition to relatively minor $w$ and $(a-B)$ ions $[21,34]$. It has been proposed that complementary $c$ and $y$ ion pairs result from direct cleavage of the 5' $\mathrm{P}-\mathrm{O}$ bond [39]. Thus, neutral nucleobase loss is not expected for RNA, in contrast to CAD and IRMPD of DNA, which proceeds through a base loss intermediate [40]. Similarly, no base loss, [M - B], ions are observed in ECD of $A_{6}$.

The observed product ions are quite different from ECD of the DNA $\mathrm{dA}_{6}$, which mainly resulted in $d / w$ type ions but no $b / y$ or $c / x$ ions [29]. This drastically different fragmentation behavior is presumably due to different preferred fragmentation pathways as a result of the hydroxyl group of the 2 ' sugar position.

\section{Electron Capture Dissociation of $C_{6}$ Dications}

Figure 2 shows the mass spectrum resulting from ECD of $\mathrm{C}_{6}$. The result is markedly different from that of $\mathrm{A}_{6}$ (Figure 1) in that only two $d / w$ and $\left(d / w+\mathrm{H}_{2} \mathrm{O}\right)$ ions are observed and no $b / y$ or $c / x$ ions are present. Very limited backbone cleavage is seen, and full sequence coverage cannot be obtained from the detected product ions. The abundance of the charge reduced nondissociated species is much lower than for $A_{6}$. The different dissociation behavior of $C_{6}$ compared with $A_{6}$ suggests that the ECD cleavage mechanism for RNA involves the nucleobases. Because the nucleobases are the most likely sites of protonation, one can envision electron capture being preferred at those locations, creating a radical site that can progress to ultimately result in cleavage of the phosphodiester backbone. Electron capture at DNA nucleobases has been shown to be energetically favored over direct capture by $\mathrm{P}=\mathrm{O}$ bonds for near zero $\mathrm{eV}$ electrons interacting with neutral DNA
[41]. We argue that this preference should be even stronger in the presence of nucleobase protonation. The same authors showed that electron attachment to nucleobases can cause backbone $\mathrm{C}-\mathrm{O}$ bond cleavage, i.e., the analog of $a / w$ or $d / z$-type cleavage, as previously proposed by Sanche and coworkers as an important route to DNA damage [42]. The different behavior for $\mathrm{A}_{6}$, i.e., the dominance of $b / y$ and $c / x$ ions, may be related to its propensity for intramolecular hydrogen bonding between the nucleobases and the phosphate backbone, which could promote alternate radical migration as compared with $\mathrm{C}_{6}$. Possibly, alternate cleavage would also be observed for the same oligonucleotide by varying the electron energy, similar to theoretical results obtained for neutral DNA [41, 42]. However, because we are operating in the "multi-pass" ECD regime, i.e., electrons are reflected through the ICR cell and can therefore cool to the optimum energy for capture [43], such experiments are difficult to perform in our current configuration.

Abundant $\left(d / w+\mathrm{H}_{2} \mathrm{O}\right)$ ions were also found in ECD of the oligodeoxynucleotide $\mathrm{dC}_{6}$, and were proposed to be formed from a pentavalent phosphorane intermediate involving the $5^{\prime}$ hydroxyl group [28]. Such a rearrangement does not involve the $2^{\prime}$ position of the sugar and can therefore also be envisioned for RNA.

\section{Electron Capture Dissociation of CGGGGC Dications}

Doubly protonated precursor ions were not detected for $G_{6}$ or $U_{6} . U_{6}$ has a very low proton affinity [37] and guanosine oligomers can easily form quadruplexes, which can be difficult to solubilize. The $G_{6}$ hexamer may represent the worst possible scenario because it is long enough to allow quadruplex formation but short enough to not have a sufficient number of phosphate groups to aid solubility. Thus, to investigate the influence of guanine on the ECD fragmentation of RNA, we analyzed the G-rich oligonucleotide CGGGGC.

Table 1 lists the corresponding ECD products. Here, both $w / d, c / x$, and $b / y$ fragments as well as one $(w / d-$

Table 1. Product ions observed from ECD (40 ms irradiation, $-1 \mathrm{~V}$ bias voltage, 50 scans) of the RNA CGGGGC. Ions labeled $\mathrm{w}$ can also be $\mathrm{d}$ ions, ions labeled $\mathrm{b}$ can also be $\mathrm{y}$ ions, and ions labeled c can also be $\mathrm{x}$ ions

\begin{tabular}{cccc}
\hline Observed $\mathrm{m} / \mathrm{z}$ & Calculated $\mathrm{m} / \mathrm{z}$ & Assignment & Error $(\mathrm{ppm})$ \\
\hline \hline 965.1655 & 965.1655 & {$[\mathrm{M}+2 \mathrm{H}]^{2+}$} & Calibrant \\
1359.202 & 1359.201 & $w_{4}^{+}$ & 0.8 \\
1553.199 & 1553.199 & $\left(w_{5}-\mathrm{G}\right)^{+}$ & $<0.1$ \\
1625.289 & 1625.290 & $b_{4}^{+}$ & -0.9 \\
1686.238 & 1686.238 & $c_{5}^{+}$ & $<0.1$ \\
1704.249 & 1704.249 & $w_{5}^{+}$ & $<0.1$ \\
1746.258 & & unknown $^{+}$ & \\
1758.254 & & unknown & \\
1778.290 & & unknown & \\
1929.317 & 1929.324 & {$\left[\mathrm{M}+\mathrm{H}^{+}\right.$} & -3.3 \\
1930.331 & 1930.331 & {$[\mathrm{M}+2 \mathrm{H}]^{+}$} & Calibrant \\
\hline
\end{tabular}




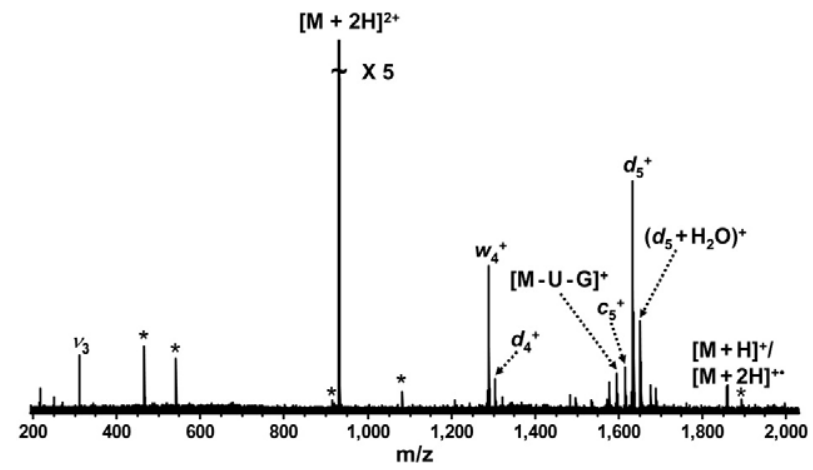

Figure 3. ECD ( $-1 \mathrm{~V}$ bias voltage, $35 \mathrm{~ms}$ irradiation, 32 scans) of the RNA GCAUAC. Electronic noise spikes are labeled with asterisks.

G) ion were observed, although the former ions dominated. The charge reduced radical species was more dominant than for $\mathrm{C}_{6}$. As for the other oligoribonucleotides, backbone cleavage was limited. The appearance of the CGGGGC ECD spectrum was more similar to the one for $\mathrm{C}_{6}$ (Figure 2) than the one for $\mathrm{A}_{6}$ (Figure 1) although no $\left(d / w+\mathrm{H}_{2} \mathrm{O}\right)$ ions were observed. Guanine, being a purine, has also been shown to be involved in extensive gas-phase intramolecular hydrogen bonding [44], which may explain the presence of $b / y$ and $c / x$ ions, as observed for $\mathrm{A}_{6}$. However, the hybrid nature of CGGGGC may result in $d / w$ ion formation as the preferred fragmentation pathway, similar to $C_{6}$.

\section{Electron Capture Dissociation of GCAUAC Cations}

To further investigate the ECD behavior of a hybrid oligoribonucleotide and also discriminate between $a$ and $z, b$ and $y, c$ and $x$, as well as $w$ and $d$ ions, the asymmetric oligoribonucleotide GCAUAC was analyzed. Figure 3 shows the corresponding ECD spectrum. Similar to the results for symmetric oligoribonucleotides, only limited backbone cleavage is observed with $d$ and $w$-type ions being dominant. No $b / y$ ions were detected in this spectrum. Thus, the precise identity of the $b / y$ ions observed above remains unclear. However, for GCAUAC, one $c$-type ion was observed, rendering its complementary $y$ ions the most probable assignment for $b / y$ ion pairs, similar to the dissociation observed in CAD and IRMPD.

\section{Electron Detachment Dissociation of $A_{6}$ Dianions}

The ECD data discussed above show that only limited backbone cleavage is observed for oligoribonucleotides, contrary to the rather extensive cleavage observed for DNA [29]. Thus, the $2^{\prime}$ hydroxyl group is likely involved in the dissociation process, consistent with a mechanism depicting initial electron capture at the nucleobases. However, for both DNA and RNA oligonucleotides, ionization in positive mode, which is re- quired for ECD, is not very efficient, and $T_{6}$ and $U_{6}$ could not be characterized. We have previously shown that negative mode ionization results in a tenfold improvement in sensitivity and that EDD can provide extensive cleavage of hexamer DNAs [32]. Thus, the oligoribonucleotides discussed above were also subjected to EDD.

An EDD spectrum of the RNA $A_{6}$ is shown in Figure 4 (top). Doubly deprotonated $\mathrm{A}_{6}$ anions were dissociated at a cathode bias voltage of $-18.1 \mathrm{~V}$ with $2 \mathrm{~s}$ irradiation time. The entire $d / w$ and $c / x$ ion series, three $b / y$, one $a / z$, one $(c / x-\mathrm{B})$, and one $\left(d / w+\mathrm{H}_{2} \mathrm{O}\right)$ ion were observed. The presence of a plentitude of sequencespecific product ions is similar to the EDD behavior of the DNA $\mathrm{dA}_{6}$ [32]. However, some differences are noted: first, we do not observe a charge reduced radical ion in EDD of $\mathrm{A}_{6}$ and only one radical product ion, [M $-\mathrm{A}]^{-*}$, is present; second, we did not observe $b / y$ or $\left(d / w+\mathrm{H}_{2} \mathrm{O}\right)$ ions in EDD of $\mathrm{dA}_{6}$; third, $c / x$ ions are more abundant in EDD of the RNA $\mathrm{A}_{6}$. We believe these differences may be rationalized by the presence of the $2^{\prime}$ hydroxyl group. A proposed mechanism for EDD of peptides involves electron detachment from deprotonated sites, e.g., backbone amide nitrogens [45]. The preferred deprotonation sites in oligonucleotides are the backbone phosphate groups. Thus, direct electron detachment from the backbone is likely although a detailed cleavage mechanism has not yet been proposed. Such a process, occurring remote from the

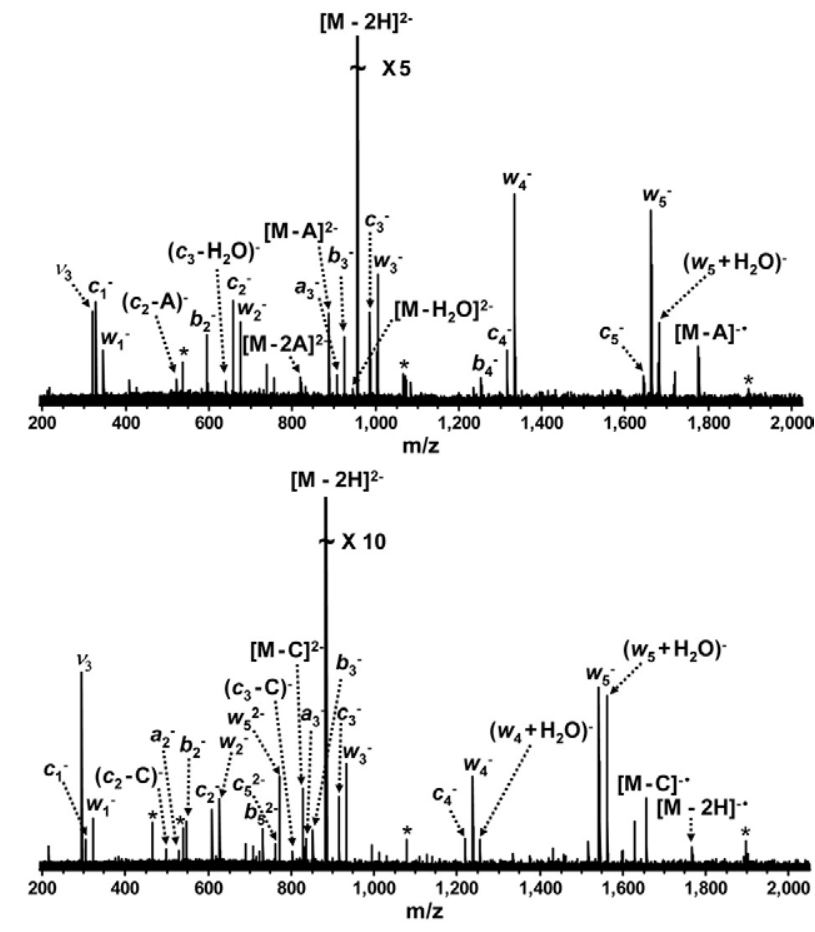

Figure 4. $\mathrm{EDD}(-18.1 \mathrm{~V}$ bias voltage, $2 \mathrm{~s}$ irradiation, 32 scans) of the RNAs $\mathrm{A}_{6}$ (top) and $\mathrm{C}_{6}$ (bottom). Complete $d / w$ and $c / x$ ion series are observed (only one of the labels is given), allowing full sequencing of the oligonucleotides. Electronic noise spikes are labeled with asterisks. 


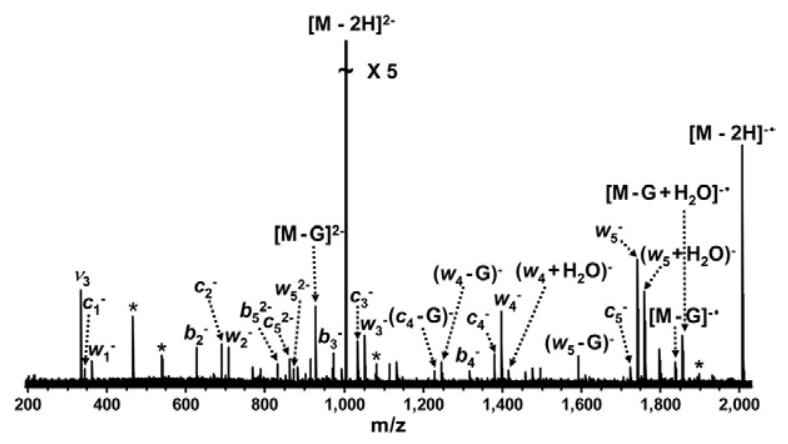

Figure 5. EDD ( $-18.3 \mathrm{~V}$ bias voltage, $2 \mathrm{~s}$ irradiation, 32 scans) of the RNA $G_{6}$. Complete $d / w$ and $c / x$ ion series are observed, allowing full sequencing of the oligonucleotide. Electronic noise spikes are labeled with asterisks.

nucleobases, rationalizes the small differences observed in EDD of various DNA sequences [32] and it is also consistent with the lower ionization energy of phosphate anions (in the range 1.16-4.57 eV [46]) compared with the most readily ionized nucleobase (guanine, 8.24 $\mathrm{eV}$ [47]). However, regardless of mechanism, the data in Figure 4, (top) demonstrate much more extensive fragmentation in EDD compared with ECD of $\mathrm{A}_{6}$. In addition, the cleavage pattern in EDD is complementary to that of CAD, IRMPD, and ECD in that $w / d$-type ions are the most abundant products rather than $c$ and $y$-type ions. Finally, the EDD data allows complete sequencing.

\section{Electron Detachment Dissociation of $\mathrm{C}_{6}$ Dianions}

Figure 4 (bottom) shows an EDD spectrum from $\mathrm{C}_{6}$. As for $\mathrm{A}_{6}$ (Figure 4 top), the entire $d / w$ and $c / x$ ion series are present as well as two complementary $a / z$ ions, three $b / y$, and two $\left(d / w+\mathrm{H}_{2} \mathrm{O}\right)$ ions. Base loss is a minor fragmentation pathway and, again, the $d / w$ ion series constitutes the dominant fragmentation channel. These data support a cleavage mechanism involving direct electron detachment from the phosphate backbone because there is no apparent nucleobase effect.

In addition to the singly charged product ions listed above, several doubly charged fragments are observed, including $c / x_{5}{ }^{2-}, b / y_{5}{ }^{2-}$, and $w / d_{5}{ }^{2-}$. Doubly charged ions are not expected in EDD of doubly charged precursor ions because the fragmentation proceeds via charge reduction [31]. However, such ions have been previously observed and were suggested to either be formed from zwitterionic precursor ions, or to be a result of direct vibrational or electronic excitation (electroninduced dissociation (EID) [48]). As for $\mathrm{A}_{6}$, more extensive backbone cleavage is observed in EDD compared with ECD and full sequence coverage is obtained.

\section{Electron Detachment Dissociation of $G_{6}$ and CGGGGC Dianions}

As mentioned above, the oligoribonucleotide $\mathrm{G}_{6}$ can be difficult to solubilize. Thus, a higher concentration (100 $\mu \mathrm{M})$ and longer accumulation time (60 s) were needed to build up sufficient signal for EDD. Figure 5 shows the EDD spectrum from $G_{6}$. As for $A_{6}$ and $C_{6}$ (Figure 4), the entire $d / w$ and $c / x$ ion series are present as well as some $b / y,\left(d / w+\mathrm{H}_{2} \mathrm{O}\right)$ and nucleobase loss fragment ions.

Base loss is more abundant for $\mathrm{G}_{6}$ than for the other oligo RNAs, consistent with previous EDD and ECD experiments of DNA and PNA, which also showed facile guanine loss [29, 32, 49]. However, guanine loss is still a minor fragmentation pathway and the $d / w$ ion series constitutes the dominant fragmentation channel.

The EDD behavior of $\mathrm{G}_{6}$ was observed to be rather sensitive to the experimental conditions, similar to ECD of $\mathrm{dA}_{6}$ [29], possibly due to variations in the gas-phase structure with more or less intramolecular hydrogen bonding. The precursor ions of CGGGGC were easier to generate than those of $\mathrm{G}_{6}$. However, their fragmentation patterns were very similar, except that no $(w / d+$ $\mathrm{H}_{2} \mathrm{O}$ ) ions were detected for CGGGGC and more abundant doubly charged product ions were observed for that oligonucleotide, consistent with the presence of cytosine.

\section{Electron Detachment Dissociation of $U_{6}$ Dianions}

Figure 6 shows the EDD spectrum from $\mathrm{U}_{6}$. As for the other RNAs (Figures 4 and 5), the entire $d / w$ ion series is present as well as some $c / x$ and $b / y$ ions. No base loss product ions are observed. One difference compared with EDD of $A_{6}, C_{6}$, and $G_{6}$ is that the abundances of $b / y$ and $c / x$ ions are comparable to those of $d / w$ ions. However, $\mathrm{U}_{6}$ is unique in that it is highly unlikely to be zwitterionic because of its low proton affinity (similar to $\mathrm{dT}_{6}$ [32]). The effect of salt bridges on the EDD mechanism is unknown but may influence the fragmentation behavior.

Because $\mathrm{U}_{6}$ is not likely to be zwitterionic, the doubly charged products $c_{5}{ }^{2-}, b_{5}{ }^{2-}$, and $w_{5}{ }^{2-}$ are likely a result of direct vibrational or electronic excitation. As for the other RNAs, full sequencing can be accomplished from the $d / w$ ion series.

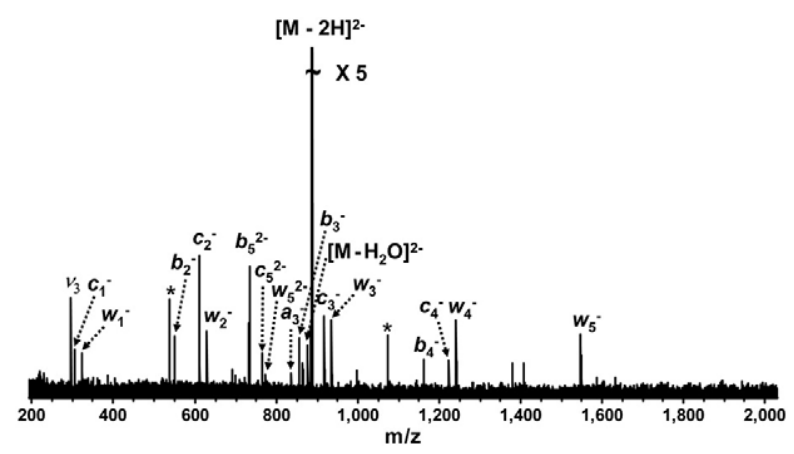

Figure 6. $\mathrm{EDD}(-18.1 \mathrm{~V}$ bias voltage, $2 \mathrm{~s}$ irradiation, 32 scans $)$ of the RNA $\mathrm{U}_{6}$. A complete $d / w$ ion series is observed, allowing full sequencing of the oligonucleotide. Electronic noise spikes are labeled with asterisks. 
Table 2. Product ions observed from EDD (2 s irradiation, $-18.2 \mathrm{~V}$ bias, 32 scans) of the RNA GCAUAC

\begin{tabular}{|c|c|c|c|}
\hline Observed $\mathrm{m} / \mathrm{z}$ & Calculated $\mathrm{m} / \mathrm{z}$ & Assignment & Error (ppm) \\
\hline 322.0433 & 322.0444 & $w_{1}^{-}$ & -3.4 \\
\hline 344.0390 & 344.0401 & $c_{1}^{-}$ & -3.2 \\
\hline 571.1315 & 571.1306 & $y_{2}^{-}$ & 1.6 \\
\hline 649.0808 & 649.0814 & $c_{2}^{-}$ & -0.9 \\
\hline 651.0997 & 651.0969 & $w_{2}^{-}$ & 4.3 \\
\hline 795.1051 & 795.1044 & $w_{5}^{2-}$ & 0.9 \\
\hline 806.1061 & 806.1022 & $c_{5}^{2-}$ & 4.8 \\
\hline 860.1172 & 860.1177 & {$[M-A]^{2-}$} & -0.6 \\
\hline 877.1580 & 877.1559 & $y_{3}^{-}$ & 2.4 \\
\hline 918.6394 & 918.6396 & {$\left[\mathrm{M}-\mathrm{H}_{2} \mathrm{O}\right]^{2-}$} & -0.2 \\
\hline 927.6449 & 927.6449 & {$[\mathrm{M}-2 \mathrm{H}]^{2-}$} & Calibrant \\
\hline 939.1186 & 939.1117 & $x_{3}^{-}$ & 7.3 \\
\hline 957.1233 & 957.1222 & $w_{3}{ }^{-}$ & 1.1 \\
\hline 978.1355 & 978.1339 & $c_{3}^{-}$ & 1.6 \\
\hline 996.1460 & 996.1445 & $d_{3}^{-}$ & 1.5 \\
\hline 1206.206 & 1206.208 & $y_{4}^{-}$ & -1.7 \\
\hline 1284.162 & 1284.159 & $\mathrm{C}_{4}^{-}$ & 2.3 \\
\hline 1286.169 & 1286.175 & $w_{4}^{-}$ & -4.7 \\
\hline 1302.183 & 1302.170 & $d_{4}^{-}$ & 10 \\
\hline 1591.211 & 1591.216 & $w_{5}^{-}$ & -3.1 \\
\hline 1625.222 & & unknown $^{-}$ & \\
\hline 1645.215 & & unknown $^{-}$ & \\
\hline 1648.234 & & unknown $^{-}$ & \\
\hline 1687.230 & & unknown $^{-}$ & \\
\hline 1631.231 & 1631.222 & $d_{5}^{-}$ & 5.5 \\
\hline 1744.240 & 1744.270 & {$[\mathrm{M}-\mathrm{U}]^{-}(?)$} & -17 \\
\hline 1855.289 & 1855.289 & {$[\mathrm{M}-2 \mathrm{H}]^{2-}$} & Calibrant \\
\hline
\end{tabular}

\section{Electron Detachment Dissociation of GCAUAC Dianions}

As for the ECD experiments, we characterized the hybrid oligoribonucleotide GCAUAC to determine the precise identity of ambiguous ion pairs. Table 2 lists the product ions observed in the corresponding EDD spectrum. Extensive backbone cleavage, including the entire $w$ ion series, three $d$-type, four $c$-type, one $x$-type, and three $y$-type ions were detected along with a couple of doubly charged products and products involving nucleobase and/or water loss. This range of fragments reveals that the most probable assignment of $b / y$ and $c / x$ ion pairs are as $y$ and $c$ ions, similar to the dissociation observed in CAD and IRMPD. By contrast, the observed $w / d$ ion pairs are likely a mixture of both $w$ and $d$-type ions, as observed in EDD of DNA.

\section{Conclusions}

This article demonstrates ECD and EDD of hexamer oligoribonucleic acids. The ECD results show a strong nucleobase dependence: $A_{6}$ generated mostly $c$ and $y$-type ions, similar to the fragmentation patterns observed in CAD and IRMPD, whereas $\mathrm{C}_{6}$ only yielded $d$ and $w$-type ions (similar to ECD of DNA). Other RNAs showed a mixture of those ion types. This behavior supports an ECD cleavage mechanism involving electron capture at the nucleobases, consistent with recent theoretical work for neutral DNA. Such a mechanism can be influenced by the presence of a $2^{\prime}$ hydroxyl group. However, only limited backbone cleavage was observed in ECD, precluding complete sequencing. EDD appears more promising for RNA structural characterization because more extensive backbone cleavage was observed at higher sensitivity. Here, $w$ and $d$ ion series constitute the most abundant fragmentation channels, similar to EDD of DNA. The only exception is $\mathrm{U}_{6}$ for which $w / d$-type cleavage competes with $c / y$ type cleavage. We propose that the EDD cleavage process is initiated via direct electron detachment from the deprotonated phosphate backbone. EDD provided full sequence coverage for all RNAs.

\section{Acknowledgments}

This work was supported by a research award from the American Society for Mass Spectrometry (sponsored by Thermo Electron), a starter grant from the Petroleum Research Fund, and an Elisabeth Caroline Crosby research award from the University of Michigan.

\section{References}

1. Johnston, W. K.; Unrau, P. J.; Lawrence, M. S.; Glasner, M. E.; Bartel, D. P. RNA-Catalyzed RNA Polymerization: Accurate and General RNA-Templated Primer Extension. Science 2001, 292, 1319-1325.

2. Walter, N. G.; Harris, D. A.; Pereira, M. J. B.; Rueda, D. In the Fluorescent Spotlight: Global and Local Conformational Changes of Small Catalytic RNAs. Biopolymers 2001, 61, 224-242.

3. Bevilacqua, P. C.; Brown, T. S.; Nakano, S.; Yajima, R. Catalytic Roles for Proton Transfer and Protonation in Ribozymes. Biopolymers 2004, 73, 90-109.

4. Fürtig, B.; Richter, C.; Wöhnert, J.; H., S. NMR Spectroscopy of RNA. Chem. Biochem. 2003, 4, 936-962.

5. Al-Hashimi, H. M. Dynamics-Based Amplification of RNA Function and Its Characterization by Using NMR Spectroscopy. Chem. Biochem. 2005, 6, 1506-1519.

6. Selvin, P. R. Fluorescence Resonance Energy Transfer. Methods Enzymol. 1995, 246, 6264-6268.

7. Zhuang, X. Single-Molecule RNA Science. Annu. Rev. Biophys. Biomol. Struct. 2005, 34, 399-414.

8. Limbach, P. A.; Crain, P. F.; McCloskey, J. A. Molecular Mass Measurement of Intact Ribonucleic Acids Via Electrospray Ionization Quadrupole Mass Spectrometry. J. Am. Soc. Mass Spectrom. 1995, 6, 27-39.

9. Crain, P. F.; McCloskey, J. A. Applications of Mass Spectrometry to the Characterization of Oligonucleotides and Nucleic Acids. Curr. Opin. Biotechnol. 1998, 9, 25-34.

10. Polson, A. G.; Crain, P. F.; Pomerantz, S. C.; McCloskey, J. A.; Bass, B. L. The Mechanism of Adenosine to Inosine Conversion by the DoubleStranded RNA Unwinding/Modifying Activity: A High-performance Liquid Chromatography-Mass Spectrometry Analysis. Biochemistry 1991, 30, 11507-11514.

11. Limbach, P. A.; Crain, P. F.; McCloskey, J. A. Summary: The Modified Nucleosides of RNA. Nucleic Acids Res. 1994, 22, 2183-2196.

12. Pomerantz, S. C.; McCloskey, J. A. Detection of the Common RNA Nucleoside Pseudouridine in Mixtures of Oligonucleotides by Mass Spectrometry. Anal. Chem. 2005, 77, 4697-4697.

13. Ni, J.; Pomerantz, S. C.; Rozenski, J.; Zhang, Y.; McCloskey, J. A. Interpretation of Oligonucleotide Mass Spectra for Determination of Sequence Using Electrospray Ionization and Tandem Mass Spectrometry. Anal. Chem. 1996, 68, 1989-1999.

14. Muddiman, D. C.; Smith, R. D. Sequencing and Characterization of Larger Oligonucleotides by Electrospray Ionization Fourier Transform Ion Cyclotron Resonance Mass Spectrometry. Rev. Anal. Chem. 1998, 17, $1-68$.

15. Benson, L. M.; Null, A. P.; Muddiman, D. C. Advantages of Thermococcus kodakaraenis (KOD) DNA Polymerase for PCR-Mass Spectrometry Based Analyses I. Am. Soc. Mass Spectrom. 2003, 14, 601-604.

16. Banoub, J. H.; Newton, R. P.; Esmans, E.; Ewing, D. F.; Mackenzie, G. Recent Developments in Mass Spectrometry for the Characterization of Nucleosides, Nucleotides, Oligonucleotides, and Nucleic Acids. Chem. Rev. 2005, 105, 1869-1916.

17. Hofstadler, S. A.; Sannes-Lowery, K. A.; Hannis, J. C. Analysis of Nucleic Acids by FTICR MS. Mass Spectrom. Rev. 2005, 24, 265-285.

18. Wu, J.; McLuckey, S. A. Gas-Phase Fragmentation of Oligonucleotide Ions. Int. I. Mass Spectrom. 2004, 237, 197-241.

19. Little, D. P.; Chorush, R. A.; Speir, J. P.; Senko, M. W.; Kelleher, N. L.; McLafferty, F. W. Rapid Sequencing of Oligonucleotides by HighResolution Mass-Spectrometry. J. Am. Chem. Soc. 1994, 116, 4893-4897. 
20. Little, D. P.; Aaserud, D. J.; Valaskovic, G. A.; McLafferty, F. W. Sequence Information from 42-108-mer DNAs (Complete for a 50 mer) by Tandem Mass Spectrometry. J. Am. Chem. Soc. 1996, 118, 9352-9359.

21. Sannes-Lowery, K. A.; Hofstadler, S. A. Sequence Confirmation of Modified Oligonucleotides Using IRMPD in the External Reservoir of an Electrospray Ionization Fourier Transform Ion Cyclotron Mass Spectrometer. J. Am. Soc. Mass Spectrom. 2003, 14, 825-833.

22. Sannes-Lowery, K. A.; Drader, J. J.; Griffey, R. H.; Hofstadler, S. A. Fourier Transform Ion Cyclotron Resonance Mass Spectrometry as a High Throughput Affinity Screen to Identify RNA Binding Ligands. Trends Anal. Chem. 2000, 19, 481-491.

23. McLuckey, S. A.; Van Berkel, G. J.; Glish, G. L. Tandem Mass Spectrometry of Small, Multiply-charged Oligonucleotides. J. Am. Soc. Mass Spectrom. 1992, 3, 60-70.

24. McLafferty, F. W.; Horn, D. M.; Breuker, K.; Ge, Y.; Lewis, M. A.; Cerda, B.; Zubarev, R. A.; Carpenter, B. K. Electron Capture Dissociation of Gaseous Multiply Charged Ions by Fourier Transform Ion Cyclotron Resonance. J. Am. Soc. Mass Spectrom. 2001, 12, 245-249.

25. Zubarev, R. A. Reactions of Polypeptide Ions with Electrons in the Gas Phase. Mass Spectrom. Rev. 2003, 22, 57-77.

26. Zubarev, R. A. Electron Capture Dissociation Tandem Mass Spectrometry. Curr. Opin. Biotechnol. 2004, 15, 12-16.

27. Cooper, H. J.; Håkansson, K.; Marshall, A. G. The Role of Electron Capture Dissociation in Biomolecular Analysis. Mass Spectrom. Rev. 2005, 24, 201-222.

28. Håkansson, K.; Hudgins, R. R.; Marshall, A. G.; O'Hair, R. A. J. Electron Capture Dissociation and Infrared Multiphoton Dissociation of Oligodeoxynucleotide Dications. J. Am. Soc. Mass Spectrom. 2003, 14, 23-41.

29. Schultz, K. N.; Håkansson, K. Rapid Electron Capture Dissociation of Mass-Selectively Accumulated Oligodeoxynucleotide Dications. Int. J. Mass Spectrom. 2004, 234, 123-130.

30. Horn, D. M.; Breuker, K.; Frank, A. J.; McLafferty, F. W. Kinetic Intermediates in the Folding of Gaseous Protein Ions Characterized by Electron Capture Dissociation Mass Spectrometry. J. Am. Chem. Soc. 2001, 123, 9792-9799.

31. Budnik, B. A.; Haselmann, K. F.; Zubarev, R. A. Electron Detachment Dissociation of Peptide Dianions: An Electron-Hole Recombination Phenomenon. Chem. Phys. Lett. 2001, 342, 299-302.

32. Yang, J.; Mo, J.; Adamson, J. T.; Håkansson, K. Characterization of Oligodeoxynucleotides by Electron Detachment Dissociation Fourier Transform Ion Cyclotron Resonance Mass Spectrometry Anal. Chem. 2005, 77, 1876-1882.

33. Schürch, S.; Bernal-Méndez, E.; Leumann, C. J. Electrospray Tandem Mass Spectrometry of Mixed-Sequence RNA/DNA Oligonucleotides. J. Am. Soc. Mass Spectrom. 2002, 13, 936-945.

34. Tromp, J. M.; Schurch, S. Gas-Phase Dissociation of Oligoribonucleotides and Their Analogs Studied by Electrospray Ionization Tandem Mass Spectrometry. J. Am. Soc. Mass Spectrom. 2005, 16, 1262-1268.

35. de Köning, L. J.; Nibbering, N. M. M.; van Orden, S. L.; Laukien, F. H. Mass Selection of Ions in a Fourier Transform Ion Cyclotron Resonance Trap using Correlated Harmonic Excitation Fields (CHEF). Int. J. Mass Spectrom. Ion Processes 1997, 165, 209-219.
36. Breuker, K.; Oh, H.; Cerda, B. A.; Horn, D. M.; McLafferty, F. W.Hydrogen Atom Loss in Electron Capture Dissociation: A Fourier Transform Ion Cyclotron Resonance Study with Single Isotopomeric Ubiquitin Ions. Eur. J. Mass Spectrom. 2002, 8, 177-180.

37. Green-Church, K. B.; Limbach, P. A. Mononucleotide Gas-Phase Proton Affinities as Determined by the Kinetic Method. J. Am. Soc. Mass Spectrom. 2000, 11, 24-32.

38. Green-Church, K. B.; Limbach, P. A.; Freitas, M. A.; Marshall, A. G. Gas-Phase Hydrogen/Deuterium Exchange of Positively Charged Mononucleotides by Use of Fourier-Transform Ion Cyclotron Resonance Mass Spectrometry. J. Am. Soc. Mass Spectrom. 2001, 12, 268-277.

39. Cerny, R. L.; Tomer, K. B.; Gross, M. L.; Grotjahn, L. Fast Atom Bombardment Combined with Tandem Mass Spectrometry for Determining Structures of Small Oligonucleotides Anal. Biochem. 1987, 165, 175-182.

40. Wan, K. X.; Gross, J.; Hillenkamp, F.; Gross, M. L. Fragmentation Mechanisms of Oligodeoxynucleotides Studied by H/D Exchange and Electrospray Ionization Tandem Mass Spectrometry. J. Am. Soc. Mass Spectrom. 2001, 12, 193-205.

41. Berdys, J.; Anusiewicz, I.; Skurski, P.; Simons, J. Damage to Model DNA Fragments from Very Low-Energy $(<1 \mathrm{eV})$ Electrons. J. Am. Chem. Soc. 2004, 126, 6441-6447.

42. Li, X.; Sevilla, M. D.; Sanche, L. Density Functional Theory Studies of Electron Interaction with DNA: Can Zero eV Electrons Induce Strand Breaks? J. Am. Chem. Soc. 2003, 125, 13668-13669.

43. McFarland, M. A.; Chalmers, M. J.; Quinn, J. P.; Hendrickson, C. L.; Marshall A. G. Evaluation and Optimization of Electron Capture Dissociation Efficiency in Fourier Transform Ion Cyclotron Resonance Mass Spectrometry. J. Am. Soc. Mass Spectrom. 2005, 16, 1060-1066.

44. Griffey, R. H.; Greig, M. J.; Robinson, J. M.; Laude, D. A. Gas-Phase Hydrogen-Deuterium Exchange in Phosphorothioate d(GTCAG) and d(TCGAT). Rapid Commun. Mass Spectrom. 1999, 13, 113-117.

45. Kjeldsen, F.; Silivra, O. A.; Ivonin, I. A.; Haselmann, K. F.; Gorshkov, M.; Zubarev, R. A. C- $\alpha$-C Backbone Fragmentation Dominates in Electron Detachment Dissociation of Gas-Phase Polypeptide Polyanions. Chem. Eur. J. 2005, 11, 1803-1812.

46. Wang, X. B.; Vorpagel, E. R.; Yang, X.; Wang, L. Experimental and Theoretical Investigations of the Stability, Energetics, and Structures of $\mathrm{H}_{2} \mathrm{PO}_{4}{ }^{-}, \mathrm{H}_{2} \mathrm{P}_{2} \mathrm{O}_{7}{ }^{2-}$, and $\mathrm{H}_{3} \mathrm{P}_{3} \mathrm{O}_{10}{ }^{2-}$ in the Gas Phase. J. Phys. Chem. A 2001, 105, 10468-10474.

47. Hush, N. S.; Cheung, A. S. Ionization Potentials and Donor Properties of Nucleic Acid Bases and Related Compounds. Chem. Phys. Lett. 1975, 34, 11-13.

48. Budnik, B. A.; Haselmann, K. F.; Elkin, Y. N.; Gorbach, V. I.; Zubarev, R. A. Applications of Electron-Ion Dissociation Reactions for Analysis of Polycationic Chitooligosaccharides in Fourier Transform Mass Spectrometry. Anal. Chem. 2003, 75, 5994-6001.

49. Olsen, J. V.; Haselmann, K. F.; Nielsen, M. L.; Budnik, B. A.; Nielsen, P. E.; Zubarev, R. A. Comparison of Electron Capture Dissociation and Collisionally Activated Dissociation of Polycations of Peptide Nucleic Acids. Rapid Commun. Mass Spectrom. 2001, 15, 969-974. 